\title{
Hospital Medicine and Perioperative Care: A Framework for High-Quality, High-Value Collaborative Care
}

\author{
Rachel E. Thompson, MD, MPH${ }^{1 *}$, Kurt Pfeifer, $\mathrm{MD}^{2}$, Paul J. Grant, MD³, Cornelia Taylor, MD4, Barbara Slawski, MD, MS²,
} Christopher Whinney, $\mathrm{MD}^{5}$, Laurence Wellikson, $\mathrm{MD}^{6}$, Amir K. Jaffer, MD, MBA7

\begin{abstract}
'Section of Hospital Medicine, Division of General Internal Medicine, University of Nebraska Medical Center, Omaha, Nebraska; ${ }^{2}$ Medical College of Wisconsin, Milwaukee, Wisconsin; ${ }^{3}$ University of Michigan Healthcare System, Ann Arbor, Michigan; ${ }^{4}$ Oregon Health \& Science University, Portland, Oregon; ${ }^{5}$ Cleveland Clinic, Cleveland, Ohio; ${ }^{6}$ Society of Hospital Medicine, Philadelphia, Pennsylvania; ${ }^{7}$ Rush University, Chicago, Illinois.
\end{abstract}

BACKGROUND: Hospitalists have long been involved in optimizing perioperative care for medically complex patients. In 2015, the Society of Hospital Medicine organized the Perioperative Care Work Group to summarize this experience and to develop a framework for providing optimal perioperative care.

METHODS: The work group, which consisted of perioperative care experts from institutions throughout the United States, reviewed current hospitalist-based perioperative care programs, compiled key issues in each perioperative phase, and developed a framework to highlight essential elements to be considered. The framework was reviewed and approved by the board of the Society of Hospital Medicine.
RESULTS: The Perioperative Care Matrix for Inpatient Surgeries was developed. This matrix characterizes perioperative phases, coordination, and metrics of success. Additionally, concerns and potential risks were tabulated. Key questions regarding program effectiveness were drafted, and examples of models of care were provided.

CONCLUSIONS: The Perioperative Care Matrix for Inpatient Surgeries provides an essential collaborative framework hospitalists can use to develop and continually improve perioperative care programs. Journal of Hospital Medicine 2017;12:277-282. (C) 2017 Society of Hospital Medicine
Of the 36 million US hospitalizations each year, 22\% are surgical. ${ }^{1}$ Although less frequent than medical hospitalizations, surgical hospitalizations are more than twice as costly. ${ }^{2}$ Additionally, surgical hospitalizations are on average longer than medical hospitalizations. ${ }^{2}$ Given the increased scrutiny on cost and efficiency of care, attention has turned to optimizing perioperative care. Hospitalists are well positioned to provide specific expertise in the complex interdisciplinary medical management of surgical patients.

In recent decades, multiple models of hospitalist involvement in perioperative care have evolved across the United States. ${ }^{3-19}$ To consolidate knowledge and experience and to develop a framework for providing the best care for surgical patients, the Society of Hospital Medicine organized the Perioperative Care Work Group in 2015. This framework was designed for interdisciplinary collaboration in building and strengthening perioperative care programs.

\section{METHODS}

The Society of Hospital Medicine recognized hospital medicine programs' need for guidance in developing collaborative care in perioperative medicine and appointed the

\footnotetext{
*Address for correspondence and reprint requests: Rachel E. Thompson, MD, MPH, Section of Hospital Medicine, Division of General Internal Medicine, 986435, University of Nebraska Medical Center, Omaha, NE 68198-6435; Telephone: 402-559-6779; Fax: 402-559-8873; E-mail: rachel.thompson@ unmc.edu
}

Received: July 21, 2016; Revised: September 20, 2016; Accepted: October 2, 2016

2017 Society of Hospital Medicine DOI 10.12788/jhm.2717
Perioperative Care Work Group in May 2015. Work group members are perioperative medicine experts from US medical centers. They have extensive knowledge of the literature as well as administrative and clinical experience in a variety of perioperative care models.

Topic Development. Initial work was focused on reviewing and discussing multiple models of perioperative care and exploring the roles that hospital medicine physicians have within these models. Useful information was summarized to guide hospitals and physicians in designing, implementing, and expanding patient-centric perioperative medicine services with a focus on preoperative and postoperative care. A final document was created; it outlines system-level issues in perioperative care, organized by perioperative phases.

Initial Framework. Group members submitted written descriptions of key issues in each of 4 phases: (1) preoperative, (2) day of surgery, (3) postoperative inpatient, and (4) postdischarge. These descriptions were merged and reviewed by the content experts. Editing and discussion from the entire group were incorporated into the final matrix, which highlighted (1) perioperative phase definitions, (2) requirements for patients to move to next phase, (3) elements of care coordination typically provided by surgery, anesthesiology, and medicine disciplines, (4) concerns and risks particular to each phase, (5) unique considerations for each phase, (6) suggested metrics of success, and (7) key questions for determining the effectiveness of perioperative care in an institution. All members provided final evaluation and editing.

Final Approval. The Perioperative Care Matrix for Inpatient Surgeries (PCMIS) was presented to the board of the Society of Hospital Medicine in fall 2015 and was approved 


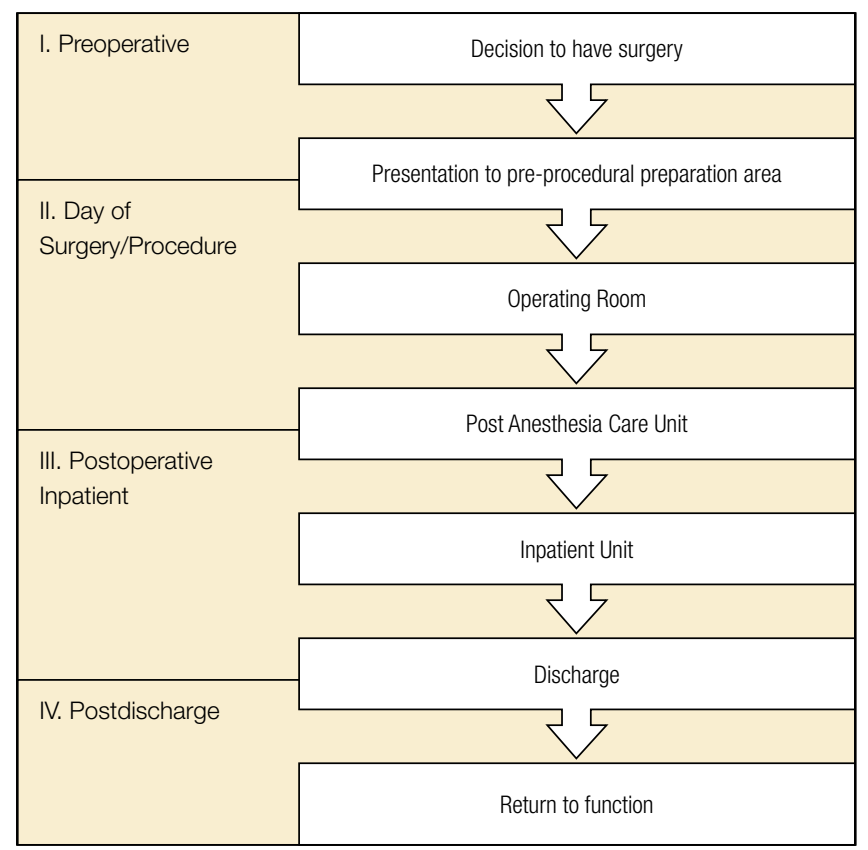

FIG. Phases of perioperative period.

for use in centering and directing discussions regarding perioperative care.

Models of Care. The Perioperative Care Work Group surveyed examples of hospitalist engagement in perioperative care and synthesized these into synopses of existing models of care for the preoperative, day-of-surgery, postoperative-inpatient, and postdischarge phases.

\section{RESULTS}

\section{Defining Key Concepts and Issues}

Hospitalists have participated in a variety of perioperative roles for more than a decade. Roles include performing indepth preoperative assessments, providing oversight to presurgical advanced practice provider assessments, providing inpatient comanagement and consultation both before and after surgery, and providing postdischarge follow-up within the surgical period for medical comorbidities.

Although a comprehensive look at the entire perioperative period is important, 4 specific phases were defined to guide this work (Figure). The phases identified were based on time relative to surgery, with unique considerations as to the overall perioperative period. Concerns and potential risks specific to each phase were considered (Table 1).

The PCMIS was constructed to provide a single coherent vision of key concepts in perioperative care (Table 2). Also identified were several key questions for determining the effectiveness of perioperative care within an institution (Table 3).

\section{Models of Care}

Multiple examples of hospitalist involvement were collected to inform the program development guidelines. The specifics noted among the reviewed practice models are described here.

Preoperative. In some centers, all patients scheduled for surgery are required to undergo evaluation at the institution's preoperative clinic. At most others, referral to the preoperative clinic is at the discretion of the surgical specialists,

TABLE 1. Concerns and Potential Risks Specific to Each Phase of Perioperative Period

\begin{tabular}{|c|c|}
\hline Phase & Concerns and Risks \\
\hline \multirow[t]{9}{*}{ Preoperative } & Inadequate or nonstandardized communication and handoffs among providers \\
\hline & Unclear or contradictory information provided to patients before surgery \\
\hline & Failed communication regarding needs for additional testing or optimization \\
\hline & Unknown or incomplete medical history from patient or in records \\
\hline & Determination that patient is not optimized \\
\hline & Determination that surgery is not best option \\
\hline & Patient needs direction regarding alternatives \\
\hline & Inability to medically optimize patient in given time —-send patient to surgery with increased risk or postpone surgery \\
\hline & Disagreement among providers as to whether patient is truly optimized \\
\hline \multirow[t]{3}{*}{ Day of surgery } & Patient not medically optimized by time of arrival in operating room \\
\hline & Identification of previously unrecognized or new medical condition that requires further evaluation \\
\hline & Several transitions occur this day—careful attention to handoffs is essential \\
\hline \multirow[t]{6}{*}{ Postoperative inpatient } & $\begin{array}{l}\text { Clinical concerns, including surgical wound (dehiscence, inadequate healing, infection), bleeding (evaluation for hemostasis, need for reoperation), pain, nausea/ } \\
\text { vomiting/constipation/ileus, venous thromboembolism, delirium, electrolyte derangement, atelectasis, hyperglycemia, hypoglycemia, anemia }\end{array}$ \\
\hline & Chronic disease decompensation \\
\hline & Unmasking of underlying disease \\
\hline & Inadequate or nonstandardized communication and handoffs among providers regarding patient's progress and ongoing needs \\
\hline & Unclear follow-up instructions, including which team member is expected to manage abnormal findings or complications \\
\hline & Situations that require change in service (surgery to medicine) or level of service (floor to intensive care unit) \\
\hline \multirow[t]{5}{*}{ Postdischarge } & Poor postoperative recovery \\
\hline & Poor coordination of care \\
\hline & Ongoing or postdischarge postoperative complications \\
\hline & Hospital readmissions \\
\hline & Triaging management of postoperative or postdischarge complications to appropriate providers (surgical vs medical) \\
\hline
\end{tabular}




\section{TABLE 2. Perioperative Care Matrix for Inpatient Surgeries ${ }^{a}$}

\begin{tabular}{|c|c|c|c|c|}
\hline & \multicolumn{4}{|c|}{ Phases in the Perioperative Continuum } \\
\hline & Preoperative & Day of Surgery & Postoperative Inpatient & Postdischarge \\
\hline $\begin{array}{l}\text { Phase } \\
\text { definitions }\end{array}$ & $\begin{array}{l}\text { From time of decision to have surgery through } \\
\text { arrival in preoperative preparation area }\end{array}$ & $\begin{array}{l}\text { From preoperative preparation, through } \\
\text { OR to PACU, to transfer to inpatient unit }\end{array}$ & $\begin{array}{l}\text { From arrival in inpatient unit (acute care floor, } \\
\text { telemetry, or ICU) through hospital discharge }\end{array}$ & $\begin{array}{l}\text { From hospital discharge through return } \\
\text { to function (includes time in nursing } \\
\text { care or rehabilitation facility until return } \\
\text { to preoperative living arrangement) }\end{array}$ \\
\hline $\begin{array}{l}\text { Requirements } \\
\text { for passing to } \\
\text { next phase }\end{array}$ & $\begin{array}{l}\text { Standardized preoperative medical assessment } \\
\text { (may differ for elective, urgent, and emergent } \\
\text { surgeries) } \\
\text { Optimization of chronic medical conditions to } \\
\text { degree possible } \\
\text { Appropriate patient and family education with } \\
\text { shared decision-making } \\
\text { Guideline-based testing, financial stewardship }\end{array}$ & $\begin{array}{l}\text { Appropriate level of care identified for } \\
\text { post-PACU (ICU vs acute care floor) } \\
\text { Clinical stability confirmed for appropriate } \\
\text { care level } \\
\text { Crucial information is communicated }\end{array}$ & $\begin{array}{l}\text { Protocol-driven early interventions focused } \\
\text { on return to function (pain control, physical/ } \\
\text { occupational therapy, bowel/bladder function) } \\
\text { Standardized approach to preventing } \\
\text { complications (eg, venous thromboembolism, } \\
\text { surgical site infection, delirium, bleeding) } \\
\text { Tailored patient/family education (anticipatory } \\
\text { guidance for recovery, management of } \\
\text { expectations) } \\
\text { As needed, assessment for home safety (per } \\
\text { physical/occupational therapy) and for skilled } \\
\text { nursing/rehabilitation facility } \\
\text { Discharge planning coordination }\end{array}$ & $\begin{array}{l}\text { Resolution of any postoperative } \\
\text { complications } \\
\text { Transfer of information back to medical } \\
\text { home and primary medical providers }\end{array}$ \\
\hline $\begin{array}{l}\text { Elements } \\
\text { of care } \\
\text { coordination } \\
\text { by discipline }\end{array}$ & $\begin{array}{l}\text { Surgical Team: determines if surgery is an } \\
\text { appropriate option, conducts consent process, } \\
\text { and provides education on surgical process/ } \\
\text { expectations } \\
\text { Anesthesiology: conducts airway assessment, } \\
\text { reviews readiness for surgery, and reviews } \\
\text { anesthesia options (may be performed day of } \\
\text { surgery) } \\
\text { Hospital Medicine: identifies medical issues } \\
\text { that affect perioperative risk, ensures } \\
\text { optimization of underlying medical conditions, } \\
\text { and provides anticipatory guidance }\end{array}$ & $\begin{array}{l}\text { Surgical Team: evaluates readiness for } \\
\text { OR and implements postoperative orders, } \\
\text { including hospital admission orders } \\
\text { Anesthesiology: evaluates readiness } \\
\text { for OR, intraoperative monitoring and } \\
\text { interventions, PACU care to ensure } \\
\text { hemodynamic stability, and adequate } \\
\text { pain control } \\
\text { Hospital Medicine: available to evaluate } \\
\text { and manage acute decompensation or } \\
\text { new medical conditions that may arise }\end{array}$ & $\begin{array}{l}\text { Surgical Team: provides general postoperative } \\
\text { care, including routine pain management, } \\
\text { surgical site care, surgery-specific } \\
\text { postoperative recovery, and discharge planning } \\
\text { Hospital Medicine: manages chronic disease, } \\
\text { acute decompensation, or new medical } \\
\text { conditions; may serve as comanager, traditional } \\
\text { consultant, or, in some cases, primary service } \\
\text { Anesthesiology: follows up intraoperative } \\
\text { or PACU complications, provides advanced } \\
\text { postoperative pain management, and, in some } \\
\text { settings, provides intensive care in surgical } \\
\text { intensive care unit }\end{array}$ & $\begin{array}{l}\text { Surgical Team: postoperative follow-up } \\
\text { appointment } \\
\text { Hospital Medicine: ensures appropriate } \\
\text { medical follow-up, as needed, by } \\
\text { primary care physician, skilled nursing } \\
\text { facility team, or hospital medicine } \\
\text { postdischarge clinic }\end{array}$ \\
\hline $\begin{array}{l}\text { Metrics of } \\
\text { success }\end{array}$ & $\begin{array}{l}\text { Adequate patient preparation (patient report, } \\
\text { measures of patient understanding) } \\
\text { Use of preoperative services } \\
\text { Timely scheduling of preoperative clinic } \\
\text { appointment } \\
\text { Clinic efficiency (no-show rates, information } \\
\text { flow) } \\
\text { Judicious preoperative testing (no unnecessary } \\
\text { tests) } \\
\text { Patient-reported experience } \\
\text { Surgeon/provider satisfaction }\end{array}$ & $\begin{array}{l}\text { OR efficiency (turnover time, time from } \\
\text { patient presentation to OR start) } \\
\text { OR delays and cancellations } \\
\text { OR complications } \\
\text { Reintubation rate } \\
\text { Floor transfer to ICU within } 24 \text { hours of } \\
\text { surgery } \\
\text { Patient-reported experience }\end{array}$ & $\begin{array}{l}\text { Complication rates } \\
\text { Rate of unanticipated return to OR } \\
\text { Length of stay (observed-to-expected ratio) } \\
\text { Mortality index } \\
\text { Surgical Care Improvement Project and Value- } \\
\text { Based Purchasing metrics } \\
\text { University HealthSystem Consortium patient } \\
\text { safety indicators } \\
\text { Patient satisfaction } \\
\text { Clarity of patient education and discharge } \\
\text { instructions (patient report, measures of patient } \\
\text { understanding) }\end{array}$ & $\begin{array}{l}\text { 30-day rate of readmissions (related } \\
\text { and unrelated) } \\
\text { Postacute care needs and expenses } \\
\text { Surgery-specific metric (infection rate) } \\
\text { Timeliness of return to prior physical } \\
\text { function and/or return to work }\end{array}$ \\
\hline
\end{tabular}

aThe perioperative care continuum encompasses care from the time the decision is made to have surgery to the time function returns after surgery. A myriad of transitions and complicated handoffs among several disciplines is involved in every phase. Thus, it is imperative to have clear plans for coordination of and communication about services throughout the care continuum. This matrix is structured by phases of the perioperative continuum. It outlines requirements for passing from one phase to the next, elements of care coordination, potential risks, unique considerations, and metrics associated with each phase. It concludes with suggested key questions that assist in designing perioperative programs.

NOTE: Abbreviations: ICU, intensive care unit; OR, operating room; PACU, postanesthesia care unit.

who have been informed of the clinic's available resources. Factors determining whether a patient has an in-person clinic visit, undergoes a telephone-based medical evaluation, or has a referral deferred to the primary care physician (PCP) include patient complexity and surgery-specific risk. Patients who have major medical comorbidities (eg, chronic lung or heart disease) or are undergoing higher risk procedures (eg, those lasting $>1$ hour, laparotomy) most often undergo a formal clinic evaluation. Often, even for a patient whose preoperative evaluation is completed by a PCP, the preoperative nursing staff will call before surgery to provide instructions and to confirm that preoperative planning is complete. Confirmation includes ensuring that the surgery consent and preoperative history and physical examination documents are in the medical record, and that all recom- mended tests have been performed. If deficiencies are found, surgical and preoperative clinic staff are notified.

During a typical preoperative clinic visit, nursing staff complete necessary regulatory documentation requirements and ensure that all items on the preoperative checklist are completed before day of surgery. Nurses or pharmacists perform complete medication reconciliation. For medical evaluation at institutions with a multidisciplinary preoperative clinic, patients are triaged according to comorbidity and procedure. These clinics often have anesthesiology and hospital medicine clinicians collaborating with interdisciplinary colleagues and with patients' longitudinal care providers (eg, PCP, cardiologist). Hospitalists evaluate patients with comorbid medical diseases and address uncontrolled conditions and newly identified symptomatology. Additional testing is 
TABLE 3. Key Questions for Determining Effectiveness of Perioperative Care in an Institution

\begin{tabular}{|c|c|}
\hline Phase & Key Questions \\
\hline \multirow[t]{5}{*}{ Preoperative } & How extensive a preoperative evaluation is medically necessary, and how is that determined? \\
\hline & $\begin{array}{l}\text { What is the current process for preparing patients for surgery? Who is involved or should be involved? How will underlying medical issues be assessed and } \\
\text { optimized? }\end{array}$ \\
\hline & How is patient care coordinated, and how is communication ensured among providers? How are disagreements managed? \\
\hline & $\begin{array}{l}\text { What is the rate or frequency of last-minute operating room cancellations and delays? What strategies are in place to optimize the preoperative process? How } \\
\text { else might the institution measure the value of preoperative assessments? }\end{array}$ \\
\hline & $\begin{array}{l}\text { What planning can and should be done in anticipation of the perioperative course? How is this plan communicated to the patient, family members, } \\
\text { and providers? }\end{array}$ \\
\hline \multirow[t]{4}{*}{ Day of surgery } & $\begin{array}{l}\text { How will pertinent information from the operating room be transferred to the intensive care unit or the acute care floor? } \\
\text { Is there a need for inpatient comanagement? How will this be determined? }\end{array}$ \\
\hline & $\begin{array}{l}\text { What clinical conditions or situations determine whether the hospital medicine team or the surgical team should provide primary services once the patient is out } \\
\text { of the operating room? }\end{array}$ \\
\hline & For urgent cases, what methods are used to address preoperative medical stability and to implement risk reduction strategies? \\
\hline & $\begin{array}{l}\text { Who is responsible for immediate care or triage when a new or an acute medical issue is identified during preoperative preparation or in the postanesthesia care } \\
\text { unit? }\end{array}$ \\
\hline \multirow[t]{6}{*}{ Postoperative inpatient } & What process is or should be in place for accurate and efficient communication and handoffs? \\
\hline & $\begin{array}{l}\text { What are the provider roles after surgery? How can the surgical team, anesthesiology, and medicine best collaborate and coordinate to provide optimal inpatient } \\
\text { care? }\end{array}$ \\
\hline & Regarding length of stay for the top 10 surgical procedures, how well does this hospital compare with its "peer" hospitals? \\
\hline & What other performance metrics are in most need of refinement? \\
\hline & $\begin{array}{l}\text { How can discharge planning begin in the preoperative phase and be coordinated across the perioperative period? What elements of transition programs (eg, } \\
\text { BOOST [Better Outcomes by Optimizing Safe Transitions]) should be incorporated, and how? }\end{array}$ \\
\hline & What effective and innovative inpatient postoperative processes has the institution endorsed and actively supported? \\
\hline \multirow[t]{4}{*}{ Postdischarge } & What standardized process and protocols are needed for communication and handoffs among providers? \\
\hline & $\begin{array}{l}\text { What are the provider roles after surgery? How can the surgical team, anesthesiology, and medicine best collaborate and coordinate to provide } \\
\text { optimal patient care? }\end{array}$ \\
\hline & What instructions are provided to the patient? How and by whom? \\
\hline & What is the 30-day readmission rate for surgical patients? What are common causes? How can these be anticipated and prevented? \\
\hline
\end{tabular}

determined by evidence- and guideline-based standards. Patients receive preoperative education, including simple template-based medication management instructions. Perioperative clinicians follow up on test results, adjust therapy, and counsel patients to optimize health in preparation for surgery.

Patients who present to the hospital and require urgent surgical intervention are most often admitted to the surgical service, and hospital medicine provides timely consultation for preoperative recommendations. At some institutions, protocols may dictate that certain surgical patients (eg, elderly with hip fracture) are admitted to the hospital medicine service. In these scenarios, the hospitalist serves as the primary inpatient care provider and ensures preoperative medical optimization and coordination with the surgical service to expedite plans for surgery.

Day of Surgery. On the day of surgery, the surgical team verifies all patient demographic and clinical information, confirms that all necessary documentation is complete (eg, consents, history, physical examination), and marks the surgical site. The anesthesia team performs a focused review and examination while explaining the perioperative care plan to the patient. Most often, the preoperative history and physical examination, completed by a preoperative clinic provider or the patient's PCP, is used by the anesthesiologist as the basis for clinical assessment. However, when information is incomplete or contradictory, surgery may be delayed for further record review and consultation.
Hospital medicine teams may be called to the pre-anesthesia holding area to evaluate acute medical problems (eg, hypertension, hyperglycemia, new-onset arrhythmia) or to give a second opinion in cases in which the anesthesiologist disagrees with the recommendations made by the provider who completed the preoperative evaluation. In either scenario, hospitalists must provide rapid service in close collaboration with anesthesiologists and surgeons. If a patient is found to be suff-ciently optimized for surgery, the hospitalist clearly documents the evaluation and recommendation in the medical record. For a patient who requires further medical intervention before surgery, the hospitalist often coordinates the immediate disposition (eg, hospital admission or discharge home) and plans for optimization in the timeliest manner possible.

Occasionally, hospitalists are called to evaluate a patient in the postanesthesia care unit (PACU) for a new or chronic medical problem before the patient is transitioned to the next level of care. At most institutions, all PACU care is provided under the direction of anesthesiology, so it is imperative to collaborate with the patient's anesthesiologist for all recommendations. When a patient is to be discharged home, the hospitalist coordinates outpatient follow-up plans for any medical issues to be addressed postoperatively. Hospitalists also apply their knowledge of the limitations of non-intensive care unit hospital care to decisions regarding appropriate triage of patients being admitted after surgery. 
Postoperative Inpatient. Hospitalists provide a $24 / 7 \mathrm{mod}$ el of care that deploys a staff physician for prompt assessment and management of medical problems in surgical patients. This care can be provided as part of the duties of a standard hospital medicine team or can be delivered by a dedicated perioperative medical consultation and comanagement service. In either situation, the type of medical care, comanagement or consultation, is determined at the outset. As consultants, hospitalists provide recommendations for medical care but do not write orders or take primary responsibility for management. Comanagement agreements are common, especially for orthopedic surgery and neurosurgery; these agreements delineate the specific circumstances and responsibilities of the hospitalist and surgical teams. Indications for comanagement, which may be identified during preoperative clinic evaluation or on admission, include uncontrolled or multiple medical comorbidities or the development of nonsurgical complications in the perioperative period. In the comanagement model, care of most medical issues is provided at the discretion of the hospitalist. Although this care includes order-writing privileges, management of analgesics, wounds, blood products, and antithrombotics is usually reserved for the surgical team, with the hospitalist only providing recommendations. In some circumstances, hospitalists may determine that the patient's care requires consultation with other specialists. Although it is useful for the hospitalist to speak directly with other consultants and coordinate their recommendations, the surgical service should agree to the involvement of other services.

In addition to providing medical care throughout a patient's hospitalization, the hospitalist consultant is crucial in the discharge process. During the admission, ideally in collaboration with a pharmacist, the hospitalist reviews the home medications and may change chronic medications. The hospitalist may also identify specific postdischarge needs of which the surgical team is not fully aware. These medical plans are incorporated through shared responsibility for discharge orders or through a reliable mechanism for ensuring the surgical team assumes responsibility. Final medication reconciliation at discharge, and a plan for prior and new medications, can be formulated with pharmacy assistance. Finally, the hospitalist is responsible for coordinating medically related hospital follow-up and handover back to the patient's longitudinal care providers. The latter occurs through inclusion of medical care plans in the discharge summary completed by the surgical service and, in complex cases, through direct communication with the patient's outpatient providers.

For some patients, medical problems eclipse surgical care as the primary focus of management. Collaborative discussion between the medical and surgical teams helps determine if it is more appropriate for the medical team to become the primary service, with the surgical team consulting. Such triage decisions should be jointly made by the attending physicians of the services rather than by intermediaries.

Postdischarge. Similar to their being used for medical problems after hospitalization, hospitalist-led postdischarge and extensivist clinics may be used for rapid follow-up of medical concerns in patients discharged after surgical admissions. A key benefit of this model is increased availability over what primary care clinics may be able to provide on short notice, particularly for patients who previously did not have a PCP. Additionally, the handover of specific follow-up items is more streamlined because the transition of care is between hospitalists from the same institution. Through the postdischarge clinic, hospitalists can provide care through either clinic visits or telephone-based follow-up. Once a patient's immediate postoperative medical issues are fully stabilized, the patient can be transitioned to long-term primary care follow-up.

\section{DISCUSSION}

The United States is focused on sensible, high-value care. Perioperative care is burgeoning with opportunities for improvement, including reducing avoidable complications, developing systems for early recognition and treatment of complications, and streamlining processes to shorten length of stay and improve patient experience. The PCMIS provides the needed platform to catalyze detailed collaborative work between disciplines engaged in perioperative care.

As average age and level of medical comorbidity increase among surgical patients, hospitalists will increasingly be called on to assist in perioperative care. Hospitalists have long been involved in caring for medically complex surgical patients, through comanagement, consultation, and preoperative evaluations. As a provider group, hospitalists have comprehensive skills in quality and systems improvement, and in program development across hospital systems nationwide. Hospitalists have demonstrated their value by focusing on improving patient outcomes and enhancing patient engagement and experiences. Additionally, the perioperative period is fraught with multiple and complicated handoffs, a problem area for which hospital medicine has pioneered solutions and developed unique expertise. Hospital medicine is well prepared to provide skilled and proven leadership in the timely development, improvement, and expansion of perioperative care for this increasingly older and chronically ill population.

Hospitalists are established in multiple perioperative roles for high-risk surgical patients and have the opportunity to expand optimal patient-centric perioperative care systems working in close concert with surgeons and anesthesiologists. The basics of developing these systems include (1) assessing risk for medical complications, (2) planning for perioperative care, (3) developing programs aimed at risk reduction for preventable complications and early identification and intervention for unavoidable complications, and (4) guiding quality improvement efforts, including planning for frequent handoffs and transitions.

As a key partner in developing comprehensive programs in perioperative care, hospital medicine will continue to shape the future of hospital care for all patients. The PC- 
MIS, as developed with support from the Society of Hospital Medicine, will aid efforts to achieve the best perioperative care models for our surgical patients.

Disclosures: Financial activities outside the submitted work: Drs. Pfeifer and Jaffer report payment for development of educational presentations; Dr. Grant reports payment for expert testimony pertaining to hospital medicine; Drs. Grant and Jaffer report royalties from publishing; Drs. Thompson, Pfiefer, Grant, Slawski, and Jaffer report travel expenses for speaking and serving on national committees; and Drs. Slawski and Jaffer serve on the board of the Society of Perioperative Assessment and Quality Improvement. The other authors have nothing to report.

\section{References}

1. Colby SL, Ortman JM. Projections of the Size and Composition of the U.S. Popula tion: 2014 to 2060 (Current Population Reports, P25-1143). Washington, DC US Census Bureau; 2014. https://www.census.gov/content/dam/Census/library/publications/2015/demo/p25-1143.pdf. Published March 2015. Accessed May 26, 2016.

2. Steiner C, Andrews R, Barrett M, Weiss A. HCUP Projections: Cost of Inpatient Discharges 2003 to 2013 (Rep 2013-01). Rockville, MD: US Dept of Health and Human Services, Agency for Healthcare Research and Quality; 2013. http://www. hcup-us.ahrq.gov/reports/projections/2013-01.pdf. Published December 11, 2013. Accessed May 26, 2016.

3. Auerbach AD, Wachter RM, Cheng HQ, et al. Comanagement of surgical patients between neurosurgeons and hospitalists. Arch Intern Med. 2010;170(22): 2004-2010.

4. Batsis JA, Phy MP, Melton LJ 3rd, et al. Effects of a hospitalist care model on mortality of elderly patients with hip fractures. J Hosp Med. 2007;2(4):219-225.

5. Carr AM, Irigoyen M, Wimmer RS, Arbeter AM. A pediatric residency experience with surgical co-management. Hosp Pediatr. 2013;3(2):144-148.

6. Della Rocca GJ, Moylan KC, Crist BD, Volgas DA, Stannard JP, Mehr DR. Comanagement of geriatric patients with hip fractures: a retrospective, controlled, cohort study. Geriatr Orthop Surg Rehabil. 2013;4(1):10-15.
7. Fisher AA, Davis MW, Rubenach SE, Sivakumaran S, Smith PN, Budge MM. Outcomes for older patients with hip fractures: the impact of orthopedic and geriatric medicine cocare. J Orthop Trauma. 2006;20(3):172-178.

8. Friedman SM, Mendelson DA, Kates SL, McCann RM. Geriatric co-management of proximal femur fractures: total quality management and protocol-driven care result in better outcomes for a frail patient population. J Am Geriatr Soc. 2008;56(7):1349-1356.

9. Huddleston JM, Long KH, Naessens JM, et al; Hospitalist-Orthopedic Team Trial Investigators. Medical and surgical comanagement after elective hip and knee arthroplasty: a randomized, controlled trial. Ann Intern Med. 2004;141(1):28-38.

10. Mendelson DA, Friedman SM. Principles of comanagement and the geriatric fracture center. Clin Geriatr Med. 2014;30(2):183-189.

11. Merli GJ. The hospitalist joins the surgical team. Ann Intern Med. 2004;141(1): 67-69.

12. Phy MP, Vanness DJ, Melton LJ 3rd, et al. Effects of a hospitalist model on elderly patients with hip fracture. Arch Intern Med. 2005;165(7):796-801.

13. Pinzur MS, Gurza E, Kristopaitis T, et al. Hospitalist-orthopedic co-management of high-risk patients undergoing lower extremity reconstruction surgery. Orthopedics. 2009;32(7):495.

14. Rappaport DI, Adelizzi-Delany J, Rogers KJ, et al. Outcomes and costs associated with hospitalist comanagement of medically complex children undergoing spinal fusion surgery. Hosp Pediatr. 2013;3(3):233-241.

15. Rappaport DI, Cerra S, Hossain J, Sharif I, Pressel DM. Pediatric hospitalist preoperative evaluation of children with neuromuscular scoliosis. J Hosp Med. 2013;8(12):684-688

16. Roy A, Heckman MG, Roy V. Associations between the hospitalist model of care and quality-of-care-related outcomes in patients undergoing hip fracture surgery. Mayo Clin Proc. 2006;81(1):28-31.

17. Sharma G, Kuo YF, Freeman J, Zhang DD, Goodwin JS. Comanagement of hospitalized surgical patients by medicine physicians in the United States. Arch Intern Med. 2010;170(4):363-368

18. Simon TD, Eilert R, Dickinson LM, Kempe A, Benefield E, Berman S. Pediatric hospitalist comanagement of spinal fusion surgery patients. J Hosp Med. 2007;2(1):23-30

19. Whinney C, Michota F. Surgical comanagement: a natural evolution of hospitalist practice. J Hosp Med. 2008;3(5):394-397. 